\title{
Supercritical antisolvent precipitation of PHBV microparticles
}

\author{
Mariana Sousa Costa ${ }^{a}$, Ana Rita C. Duarte ${ }^{b}, *$ M. Margarida Cardoso ${ }^{a}$, Catarina M.M. Duarte $^{b}$ \\ ${ }^{a}$ REQUIMTE/CQFB, Departamento de Química, FCT-UNL, 2829, 516 Caparica, Portugal \\ ${ }^{\mathrm{b}}$ Nutraceuticals and Delivery Laboratory, ITQB/IBET, Aptd. 12, 2781-901 Oeiras, Portugal \\ Received 5 May 2006; received in revised form 28 July 2006; accepted 5 August 2006 \\ Available online 12 August 2006
}

\begin{abstract}
The micronization of poly(3-hydroxybutyrate-co-3-hydroxyvalerate) (PHBV) from organic solutions using supercritical antisolvent (SAS) technique has been successfully achieved.

SAS experiments were carried out at different operational conditions and microspheres with mean diameters ranging from 3 to $9 \mu \mathrm{m}$ were obtained. The effect of $\mathrm{CO}_{2}$ and liquid flow, temperature and pressure on particle size and particle size distribution was evaluated. The microspheres were precipitated from a dichloromethane (DCM) solution. The best process conditions for this mixture were, according to our study, $40^{\circ} \mathrm{C}, 100 \mathrm{bar}$, $1 \mathrm{~mL} \mathrm{~min}^{-1}$ liquid flow and $10 \mathrm{~L} \mathrm{~min}^{-1}$ carbon dioxide flow.

Experiments with polymers containing different HV percentages were carried out. The powders obtained became more spherical as the HV content decreased.
\end{abstract}

(C) 2006 Elsevier B.V. All rights reserved.

Keywords: Supercritical fluids; Micronization; SAS; Natural polymers; PHBV; Drug delivery

\section{Introduction}

The use of biocompatible and biodegradable polymers as drug-carrier systems is increasing continuously in pharmaceutical applications, since they can successfully deliver the drug to a target side that reduces the side effects and enhance the therapeutic benefit (Shariati and Peters, 2003). Several controlled drug delivery systems are available and can be used however the use of micro and nanoparticles appears to be the most interesting way as these systems facilitate the diffusion through biological barriers. For such systems, size and morphology of the polymer matrix assumes an extremely important role in the drug release and pharmacokinetics. Although several polymers have been used in the pharmaceutical industry, polyhydroxyalkanoates (PHAs), a biodegradable thermoplastic polymer produced by renewable resources, have gained extensive interest as drug carrier due to its singular properties (Pouton and Akhtar, 1996; Chen and Wu, 2005). The most common type of PHAs is poly(3-hydroxybutyrate) (PHB). PHB exhibits properties similar to polypropylene (PP) such as melting point, degree

\footnotetext{
* Corresponding author. Fax: +351214461161.

E-mail address: cduarte@itqb.unl.pt (A.R.C. Duarte).
}

of cristallinity and glass transition temperature. However PHB is stiffer and more brittle than PP, which can restrict its range of applications. On the other hand, PHB copolymers with 3-hydroxyvalerate (PHBV) are less stiff and tougher (Gunaratne and Shanks, 2005; Ray and Bousmina, 2005). The properties of this copolymer depend on the HV content, which determines the polymer crystallinity (Peng et al., 2003). The use of PHBV had a tremendous increase in applications in the biomedical field, in the past few years (Sendil et al., 1999; Baran et al., 2002; Chen and Davis, 2002; Chen and Wu, 2005), due to the fact that it is possible to prepare an appropriate controlled drug delivery system that gradually degrades in the body.

Production of micro or nanoparticles using supercritical fluid technology is very attractive since it provides an alternative solution to the various problems encountered in traditional techniques (Yeo and Kiran, 2005). The possibility of producing very small particles with a narrow size distribution using mild and inert conditions represents a major improvement over the conventional processes (Elvassore et al., 2003).

One of the available particle formation technique based on supercritical fluids is the supercritical antisolvent process, referred as SAS, ASES, PCA, or SEDS in literature (Foster et al., 2003; Yeo and Kiran, 2005) depending on the process arrange- 
ments and apparatus. Nevertheless, the principle inherent to all these processes is the same, i.e., to decrease the solvent power of the liquid by addition of an antisolvent in which the solute is insoluble. The selection of a proper combination of the organic solvent and the antisolvent for a particular polymer is essential for the success of the process. The organic solvent should have a reasonable miscibility towards the polymer and also a high solubility in the supercritical fluid under moderate operating conditions. This method of particle formation is based on two mechanisms that take place simultaneously, on one hand, the fluid penetrates into the droplets where it acts as an antisolvent for the dissolved material and on the other hand the organic solvent evaporates on the supercritical fluid so that precipitation occurs (Mukhopadhyay and Dalvi, 2004). Since the diffusivity of the supercritical fluid is higher than the diffusivity of a liquid, the diffusion of the fluid into the organic solvent produces a faster supersaturation of the solute solved in the liquid and therefore its precipitation into micronized particles.

The low solubility of polymers in carbon dioxide and their relatively high solubility in organic solvents provide suitable conditions to preferably employ this process for particle formation and design of improved controlled delivery systems.

Process variables, such as temperature, pressure and flow rate, have a strong influence in the morphology of the particles, as well as their particle size and particle size distribution. These variables directly interfere with the basic mechanisms that control particle formation, which are fluid dynamics, mass transfer, nucleation kinetics and thermodynamics (Reverchon et al., 2003).

Bleich et al. (1993) report the use of supercritical fluids, namely the ASES technique to process PHB. The aim of this work is to provide a better understanding of the process variables, such as concentration of liquid solution, pressure, temperature, liquid and carbon dioxide flow, in the precipitation of $\mathrm{PHBV}$, in order to optimise the process conditions. The effect of the HV content on the copolymer was also evaluated.

\section{Experimental procedure}

\subsection{Materials}

Poly(3-hydroxybutyrate), PHB (CAS 26063-00-3), poly(3hydroxybutyrate-co-3-hydroxyvalerate), PHBV, with $8 \%$ and $12 \%$ HV (CAS 80181-31-3) and poly[(R)-3-hydroxybutyric acid-co- $(R)$-3-hydroxyvaleric acid], PHBV, with $30 \%$ HV (CAS 92267-82-8), were purchased from Sigma, Aldrich and Fluka. Dichloromethane, DCM (CAS 78-09-2, $99.9 \%$ purity) and chloroform (CAS 67-66-3, 99.0\% purity) were purchased from Panreac. Carbon dioxide (99.998 mol\%) was supplied by Air Liquide. All chemicals were used with no further purification.

\subsection{Microsphere preparation}

The precipitation experiments were carried out in a SAS apparatus, which has been described elsewhere (Duarte et al., 2006). The apparatus works in a continuous co-current mode and it consists of a precipitator in which the antisolvent and the liquid solution are separately fed to the top of the chamber and are continuously discharged from the bottom. The liquid solution is pumped into the chamber by a high pressure piston pump (Knauer HPLC pump K-501). The antisolvent is delivered by means of a high pressure piston pump (Haskel model MCPV-71).

The precipitator is a cylindrical vessel with an inner volume of $500 \mathrm{~cm}^{3}$. The liquid solution is delivered into the chamber through a stainless steel nozzle. The carbon dioxide is heated, up to the temperature of the experiment, before entering the precipitator in a tube section by an electric cable (CS 12854), which is connected to a temperature controller (Ero Electronic LMS-491-13).

The precipitator is heated by means of two electric thin bands heater (Watlow STB3J2J1) also connected to a temperature controller. The pressure in the chamber is measured by a pressure gauge manometer (Bourdon-Haenni models MVX7 D $30 \mathrm{~B} 38$ ). The outflow is regulated by a micrometric valve (Hoke $1315 \mathrm{G} 4 \mathrm{Y}$ ) located at the bottom of the precipitator. This valve is heated with an electric cable (CS 12854) in order to prevent the freezing of carbon dioxide due to the rapid depressurisation $\left(50^{\circ} \mathrm{C}\right)$. A filter of sintered steel with $0.1 \mu \mathrm{m}$ porosity is placed at the bottom of the vessel to collect the particles produced.

The solvents are separated and recovered from a second vessel. Usually the temperature of the vessel is around $15^{\circ} \mathrm{C}$ and pressure 5-10 bar. The pressure in the separator is measured by a pressure gauge manometer (Bourdon-Haenni model MVX7 D30 B29) and regulated by a back pressure valve (Tescon europe).

The carbon dioxide flow rate and the total quantity of antisolvent used are measured by a flow meter (Aalborg GFM 17S-VADL2-E).

\subsection{Particle characterization}

\subsubsection{Scanning electron microscopy-SEM}

Samples of the precipitated powder were observed by a ZEISS 960 scanning electron microscope (SEM). The particles were fixed by mutual conductive adhesive tape on aluminium stubs and covered with gold palladium using a sputter coater.

\subsubsection{Particle size and size distribution}

The particle size and size distribution of the prepared microparticles were measured by Laser diffraction spectrometry (Coulter LS 130, Coulter Electronics). The dried powder samples were suspended in deionised water with a surfactant solution (Coulter Dispersant, Coulter) and sonicated for $1 \mathrm{~min}$ with an ultra-sound probe $(500 \mathrm{~W}$, Vibra Cell, Sonics \& Materials, Inc.) before measurement. The obtained homogeneous suspension was determined for the volume mean diameter, size distribution and polydispersity. Each sample was analysed three times.

\subsubsection{Differential scanning calorimetry}

Differential scanning calorimetry (DSC) measurements were carried out on a Setaram DSC 131 scanning calorimeter equipped with a thermal analysis data system Samples of $10 \mathrm{mg}$ 

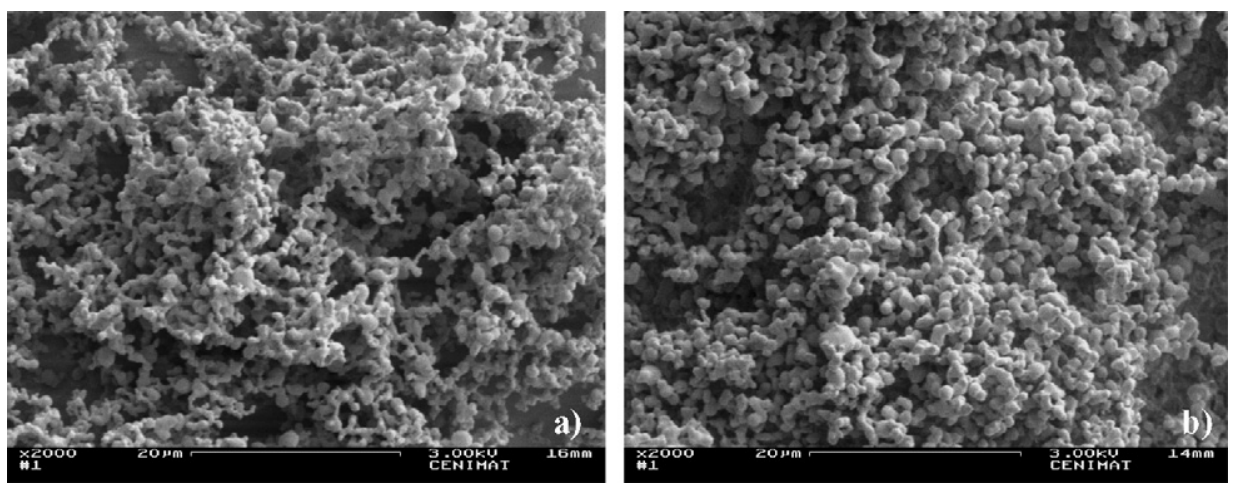

Fig. 1. PHBV micronized from (a) chloroform and (b) dicloromethane.

were placed in aluminum pans and sealed The probes were heated from 20 to $250^{\circ} \mathrm{C}$ at a rate of $10^{\circ} \mathrm{C} / \mathrm{min}$ under nitrogen atmosphere.

\section{Results and discussion}

In this study, the possibility of micronization of biocompatible and biodegradable polymers such PHB and PHBV using supercritical fluid technology is evaluated. The selection of a proper organic solvent is essential for the success of the process since the miscibility of the liquid solvent in supercritical $\mathrm{CO}_{2}$ plays a key role in this technique. Therefore, volumetric expansion curves have to be considered before starting the experiments for a given solvent. The best organic solvents for these polymers are chloroform and dichloromethane (Pouton and Akhtar, 1996). The operational conditions for the first experiments were chosen considering the volumetric expansion curves (Scurto et al., 2001; Vega-González et al., 2002), and taken into account the fact that the presence of a single phase favours the process. Therefore, a preliminary experiment using a solution of chloroform and a solution of dichloromethane were performed at 100 and $80 \mathrm{bar}$, respectively and $40^{\circ} \mathrm{C}$. This experiment was done in order to evaluate which solvent is the best one for the antisolvent micronization process. Particles, precipitated from both solutions using $\mathrm{CO}_{2}$ as an anti-solvent, present similar morphology as it can be seen on Fig. 1. Furthermore, the particles of PHBV precipitated from organic solutions through a $300 \mu \mathrm{m}$ nozzle exhibit identical narrow size distribution with a mean particle size of 5.8 and $5.3 \mu \mathrm{m}$ for dichloromethane and chloroform, respectively.

Despite the similarity of results using these two solvents, the selected organic solvent to precipitate PHBV using SAS technique was DCM due to its lower toxicity, and also because a higher yield was achieved when using this solvent.

The experiments were designed in order to determine the best operational conditions for the production of PHBV microparticles. In each set of experiments one of the variables was modified so that, the effect of the different variables on the morphology, particle size and particle size distribution could be evaluated. The experiments performed are summarized in Table 1.

The effect of the polymer concentration in the liquid solution on the particle size was evaluated. From the results of the SEM analysis (Fig. 2) it is possible to conclude that a lower concentration favours the precipitation of particles with smaller size diameter. The SEM analysis demonstrated that agglomerated particles were obtained when a higher concentration was used for the precipitation process (Fig. 2b). Therefore, all other experiments were carried out with a low concentration solution.

The influence of the temperature was the second parameter studied. Three experiments show that an isobaric increase in temperature from 35 to $40{ }^{\circ} \mathrm{C}$ causes a decrease in the mean particle size from 7.0 to $5.9 \mu \mathrm{m}$. Experiments performed at higher

Table 1

Summary of the experiments performed

\begin{tabular}{|c|c|c|c|c|c|c|c|}
\hline Experiment & System & $P$ (bar) & $T\left({ }^{\circ} \mathrm{C}\right)$ & $C_{0}\left(\mathrm{mg} \mathrm{mL}^{-1}\right)$ & Liquid flow $\left(\mathrm{mL} \mathrm{min}^{-1}\right)$ & $\mathrm{CO}_{2}$ flow $\left(\mathrm{L} \mathrm{min}^{-1}\right)$ & $\operatorname{MSD}(\mu \mathrm{m})$ \\
\hline 1 & PHBV $12 \%$ & 80 & 40 & 25 & 0.75 & 10 & - \\
\hline 2 & PHBV $12 \%$ & 80 & 40 & 12.5 & 0.75 & 10 & 5.9 \\
\hline 3 & PHBV $12 \%$ & 80 & 35 & 12.5 & 0.75 & 10 & 7.0 \\
\hline 4 & PHBV $12 \%$ & 80 & 45 & 12.5 & 0.75 & 10 & - \\
\hline 5 & PHBV $12 \%$ & 80 & 40 & 12.5 & 1.00 & 7.5 & 5.8 \\
\hline 6 & PHBV $12 \%$ & 80 & 40 & 12.5 & 1.00 & 10 & 5.9 \\
\hline 7 & PHBV $12 \%$ & 80 & 40 & 12.5 & 1.00 & 12.5 & 8.3 \\
\hline 8 & PHBV $12 \%$ & 90 & 40 & 12.5 & 1.00 & 10 & 6.9 \\
\hline 9 & PHBV $12 \%$ & 100 & 40 & 12.5 & 1.00 & 10 & 6.1 \\
\hline 10 & PHBV $8 \%$ & 80 & 40 & 12.5 & 1.00 & 10 & 7.0 \\
\hline 11 & PHBV $30 \%$ & 80 & 40 & 12.5 & 1.00 & 10 & 6.2 \\
\hline 12 & PHB & 100 & 40 & 12.5 & 1.00 & 10 & 5.9 \\
\hline
\end{tabular}





Fig. 2. Effect of concentration of the liquid solution (a) $12.5 \mathrm{mg} \mathrm{mL}^{-1}$ and (b) $25 \mathrm{mg} \mathrm{mL}^{-1}$.

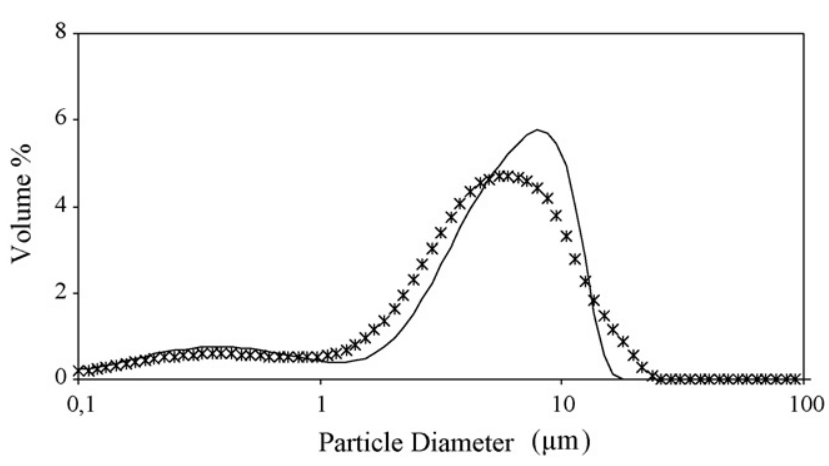

Fig. 3. Particle size distribution of PHBV micronized from a liquid solution flow of $0.75 \mathrm{~mL} \mathrm{~min}^{-1}(*)$ and $1 \mathrm{~mL} \mathrm{~min}^{-1}(-)$.

temperatures, produced a polymeric film in the bottom of the vessel. So, the optimal temperature for the precipitation of PHBV microparticles is $40^{\circ} \mathrm{C}$.

Experiments with different liquid solution and carbon dioxide flows were performed. The liquid flow does not seem to affect the particle size. Nevertheless, the particle size distribution is narrower when particles were precipitated from a liquid solution flow of $1 \mathrm{~mL} \mathrm{~min}^{-1}$ (Fig. 3). The $\mathrm{CO}_{2}$ flow varied from 7.5 to 12.5 $\mathrm{L} \mathrm{min}^{-1}$. As it can be seen from Fig. 4, an increase in carbon dioxide flow increases the particle size. Although there are no significant differences, regarding particle size and particle size distribution from experiments performed at 7.5 and $10 \mathrm{~L} \mathrm{~min}^{-1}$,



Fig. 4. Effect of $\mathrm{CO}_{2}$ flow rate on the mean particle size. the higher carbon dioxide flow was chosen to continue the experiments. This promotes a better mixing between the solvents and higher turbulence near the jet point, which favours the precipitation process. Therefore, from the results obtained, $1 \mathrm{~mL} \mathrm{~min}^{-1}$ liquid flow and $10 \mathrm{~L} \mathrm{~min}^{-1} \mathrm{CO}_{2}$ flow were chosen to continue the study.

The last variable evaluated in the precipitation process was the pressure. A pressure increase from 80 to 90 bar caused a significant decrease in the particle size, however a further increase in pressure did not significantly influenced the diameter of the particles (Fig. 5). Reverchon (Reverchon, 1999) found that for pressures larger than the asymptotic volume expansion there is no significant effect of the pressure in the particle size and particle size distribution of the powders precipitated.

With the results obtained, it is possible to conclude that for this system the best operating conditions are $40^{\circ} \mathrm{C}, 100 \mathrm{bar}$, $1 \mathrm{~mL} \mathrm{~min}^{-1}$ liquid flow and $10 \mathrm{~L} \mathrm{~min}^{-1}$ carbon dioxide flow.

Furthermore, the specific surface area was analyzed. As expected the surface area decreases with increasing particle size (Fig. 6). Small errors might derive from the presence of agglomerates that were not completely dispersed in the sample preparation.

Differential scanning calorimetry was performed in order to analyse the differences between the processed and unprocessed polymer, nevertheless no major conclusions can be withdrawn from this analysis as there is a splitting of the fusion peak.

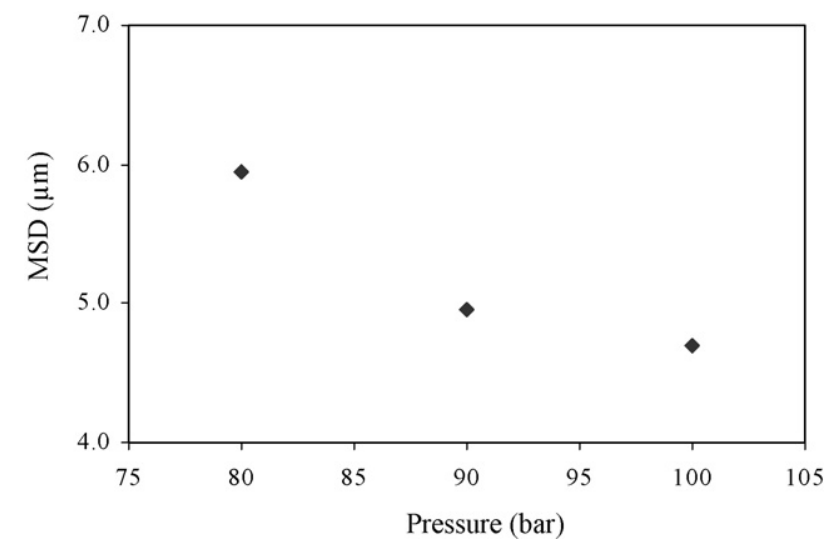

Fig. 5. Effect of pressure on the mean size diameter. 


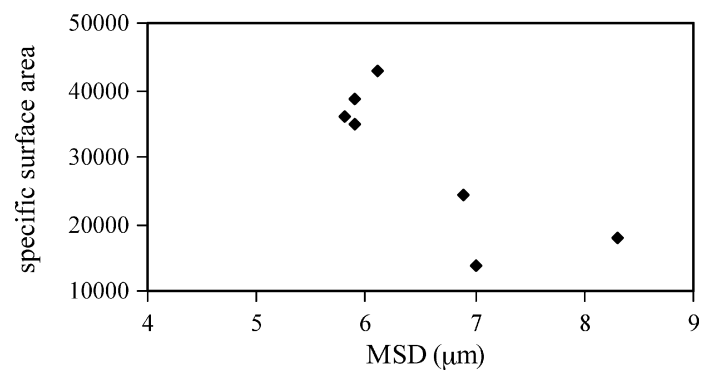

Fig. 6. Specific surface area vs. particle size.

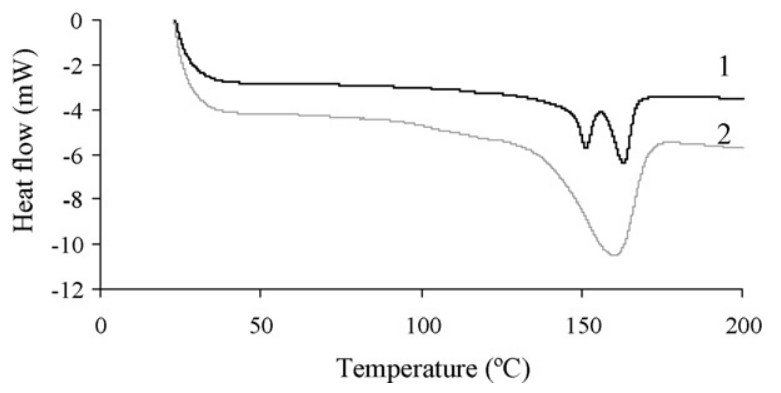

Fig. 7. Diferential scanning calorimetry (DSC) of PHBV $8 \%$ (1) processed (experiment 10) (2) unprocessed.

Fig. 7 presents the DSC for $\mathrm{PHBV} 8 \%$ processed at $40^{\circ} \mathrm{C}, 80$ bar (experiment 10) and also the unprocessed PHBV 8\%. The use of ultra-sounds to enhance the solubility of PHBV in the organic solvent might promote the break down of the polymeric chains, consequently the sample will present a broader molecular weight distribution than the initial sample. The heterogeneity of the

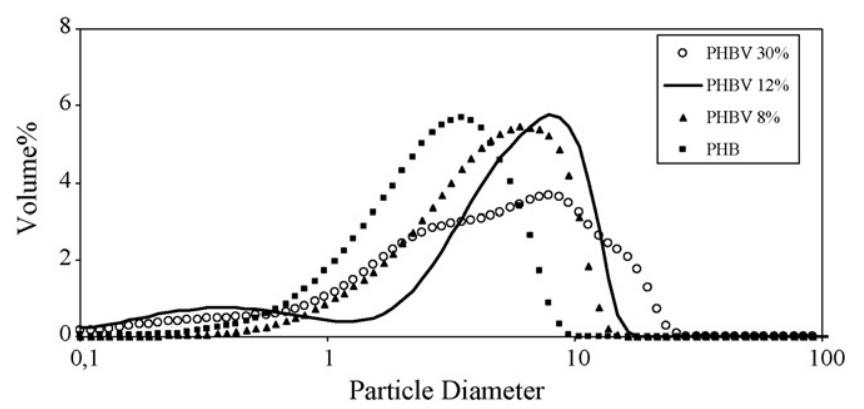

Fig. 9. Particle size distribution of PHBV 30\% (○); PHBV 12\% (-); PHBV 8\% (ム); PHB (ם).

resulting sample causes the splitting of the peak and therefore no substantial conclusions can be taken.

Experiments with polymers containing different $\mathrm{HV}$ percentages were also carried out. Fig. 8 presents the SEM images of the particles prepared from different polymer blends, confirming the success of the experiments.

There is an obvious difference in the morphology of the particles precipitated with $30 \% \mathrm{HV}$ and the others. This difference is not so evident when the $\mathrm{HV}$ content decreases from 12 or $8 \%$. In these cases the particles obtained become more spherical. The difference in the particle size distribution of the PHB particles (Fig. 9) precipitated might be due to the fact that this experiment was carried out with a different solvent and different operational conditions. The PHB had to be precipitated from a chloroform solution because it was impossible to dissolve this polymer in the same concentration using dichloromethane. For this reason, the precipitating conditions were slightly different, i.e., the pressure was 100 bar instead of 80 bar. This ensured the presence of
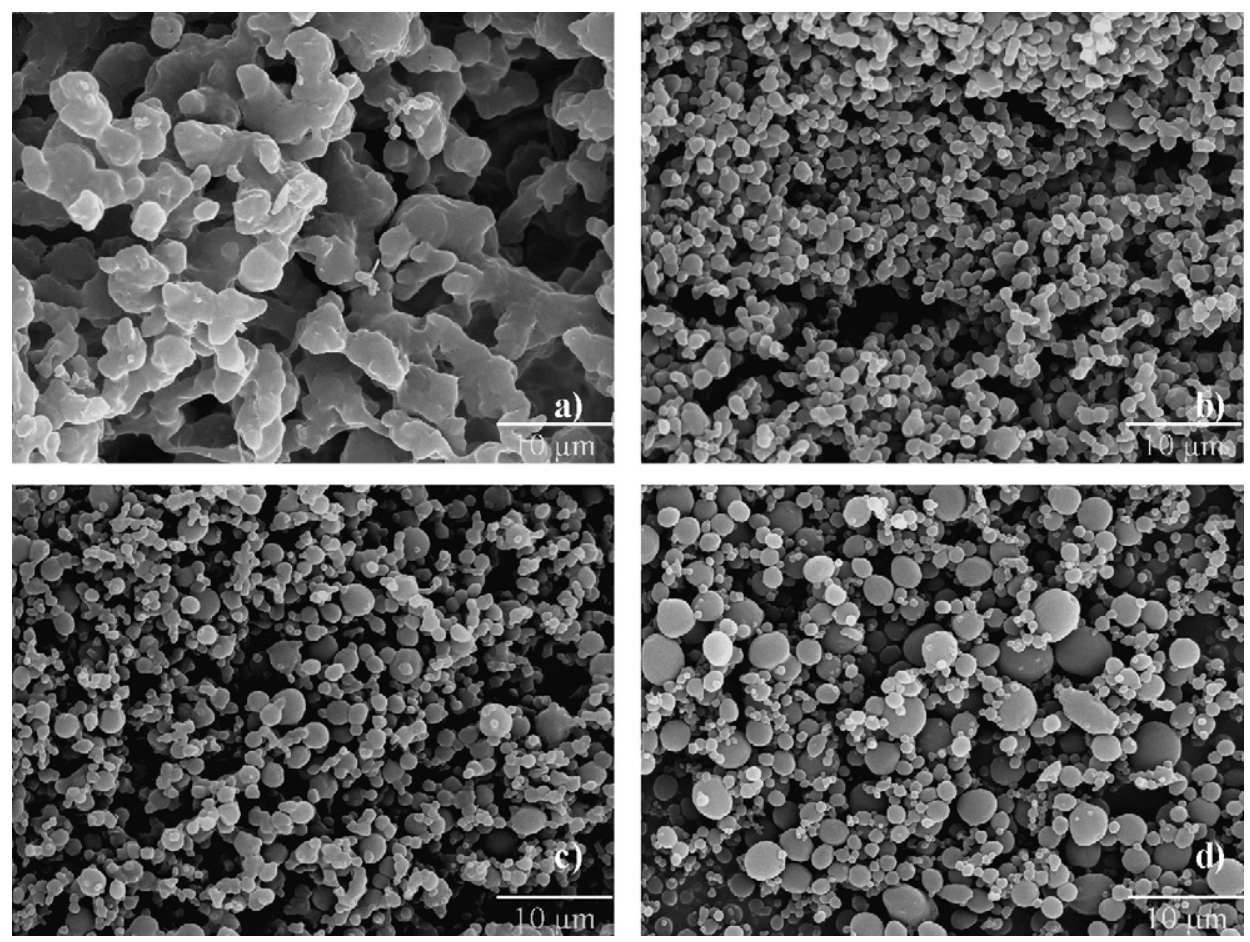

Fig. 8. SEM images of (a) PHBV 30\%; (b) PHBV 12\%; (c) PHBV 8\%; (d) PHB. 


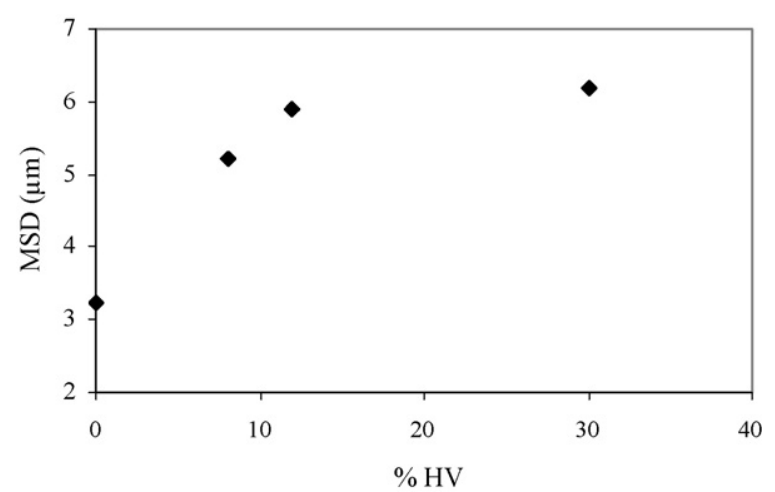

Fig. 10. Mean particle size of powder precipitated from different PHBV blends.

a single phase in the precipitation chamber. In Fig. 10, the mean particle size of the different polymers is plotted as a function of the HV content.

\section{Conclusions}

PHBV microspheres were successfully precipitated from an organic solution using the supercritical antisolvent process (SAS). Operational conditions that affect the particle size and particle size distribution, such as liquid and carbon dioxide flow, pressure and temperature, were evaluated in order to optimise the process and smaller particles were obtained at $40^{\circ} \mathrm{C}$ and 80 bar with $1 \mathrm{~mL} \mathrm{~min}^{-1}$ liquid flow and $10 \mathrm{~L} \mathrm{~min}^{-1} \mathrm{CO}_{2}$ flow rate.

Experiments with polymers with different HV content were also carried out. The powders obtained become more spherical as the HV content decreases.

\section{Acknowledgments}

Mariana Sousa Costa and Ana Rita C. Duarte are grateful for financial support from SFRH/BD/17224/2004 and SFRH/BD/10780/2002 grants, respectively. This work was financially supported by FCT-MCES, Portugal, under contract POCTI/EQU/46715/2002 from the QCA III/FEDER. The authors would like to acknowledge the Analytical Services Laboratory of REQUIMTE for the DSC results and to Prof. Ana Ramos for the transfer of knowledge related with the interpretation of DSC plots.

\section{References}

Baran, E.T., Özer, N., Hasirci, V., 2002. Poly(hydroxybutirate-cohydroxyvalerate) nanocapsules as enzyme carriers for cancer therapy: an in vitro study. J. Microencapsul. 19, 363-376.

Bleich, J., Müller, B.W., Wassmus, W., 1993. Aerosol solvent extraction system-a new microparticulate production technique. Int. J. Pharm. 97, $111-117$.

Chen, G.Q., Wu, Q., 2005. The application of polyhydroxyalcanoates as tissue engineering materials. Biomaterials 26, 6565-6578.

Chen, J., Davis, S.S., 2002. The release of diazepam from poly(hydroxybutyratehydroxyvalerate) microspheres. J. Microencapsul. 19, 191-201.

Duarte, A.R.C., Gordillo, M., Cardoso, M.M., Simplício, A.L., Duarte, C.M.M., 2006. Preparation of ethylcellulose/methylcellulose blends by supercritical antisolvent precipitation. Int. J. Pharm. 311, 50-54.

Elvassore, N., Parton, T., Bertucco, A., Di Noto, V., 2003. Kinetics of particle formation in the gas antisolvent precipitation process. AIChE J. 49, 859-868.

Foster, N., Mammucari, R., Dehghani, F., Barrett, A., Bezanehtak, K., Coen, E., Combes, G., Meure, L., Ng, A., Regtop, H.L., Tandya, A., 2003. Processing pharmaceutical compounds using dense gas technology. Ind. Eng. Chem. Res. 42, 6476-6493.

Gunaratne, L.M.W.K., Shanks, R.A., 2005. Multiple melting behaviour of poly(3-hydroxybutyrate-co-hydroxyvalerate) using step scan DSC. Eur. Polym. J. 41, 2980-2988.

Mukhopadhyay, M., Dalvi, S.V., 2004. Mass and heat transfer analysis of SAS: effects of thermodynamic states and flow rates on droplet size. J. Supercrit. Fluids 30, 333-348.

Peng, S., An, Y., Chen, C., Fei, B., Zhuang, Y., Dong, L., 2003. Isothermal crystallization of poly(3-hydroxybutyrate-co-hydroxyvalerate). Eur. Polym. J. 39, 1475-1480.

Pouton, C.W., Akhtar, S., 1996. Biosynthetic polyhydroxyalcanoates and their application in drug delivery. Adv. Drug Deliv. Rev. 18, 133-162.

Ray, S.S., Bousmina, M., 2005. Biodegradable polymers and their layered silicate nanocomposites: in greening the 21 st century materials world. Prog. Mater. Sci. 50, 962-1079.

Reverchon, E., 1999. Supercritical antisolvent precipitation of micro- and nanoparticles. J. Supercrit. Fluids 15, 1-21.

Reverchon, E., Caputo, G., De Marco, I., 2003. Role of phase behavior and atomization in the supercritical antisolvent precipitation. Ind. Eng. Chem. Res. 42, 6406-6414.

Scurto, A.M., Lubbers, C.M., Xu, G., Brennecke, J.F., 2001. Experimental measurement and modelling of the vapour-liquid equilibrium of carbon dioxide + chloroform. Fluid Phase Equilib. 190, 135-147.

Sendil, D., Gürsel, I., Wise, D.L., Hisirci, V., 1999. Antibiotic release from biodegradable PHBV microparticles. J. Control. Rel. 59, 207-217.

Shariati, A., Peters, C.J., 2003. Recent developments in particle design using supercritical fluids. Curr. Opin. Solid State Mater. Sci. 7, 371-383.

Vega-González, A., Tufeu, R., Subra, P., 2002. High-pressure vapor-liquid equilibrium for the binary systems carbon dioxide + dimethyl sulfoxide and carbon dioxide + dichloromethane. J. Chem. Eng. Data 47, 492-495.

Yeo, S.D., Kiran, E., 2005. Formation of polymer particles with supercritical fluids: a review. J. Supercrit. Fluids 34, 287-308. 